\title{
Towards quantum-limited coherent detection of terahertz waves in charge- neutral graphene
}

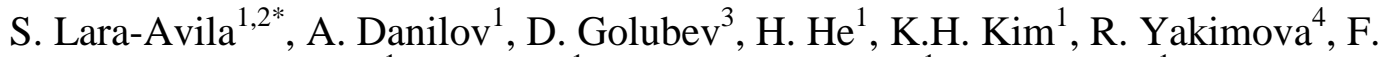 \\ Lombardi $^{1}$, T. Bauch ${ }^{1}$, S. Cherednichenko ${ }^{1}$, S. Kubatkin ${ }^{1}$ \\ ${ }^{1}$ Department of Microtechnology and Nanoscience, Chalmers University of Technology, SE-412 96, \\ Gothenburg, Sweden \\ ${ }^{2}$ National Physical Laboratory, Hampton Road, Teddington, TW11 0LW, UK \\ ${ }^{3}$ Department of Applied Physics, Aalto University, FI-00076 Aalto, Finland \\ ${ }^{4}$ Department of Physics, Chemistry and Biology, Linkoping University, S-581 83, Linköping, Sweden.
}

Spectacular advances in heterodyne astronomy with both the Herschel Space Observatory $^{1}$ and Stratospheric Observatory for Far Infrared Astronomy (SOFIA) ${ }^{2}$ have been largely due to breakthroughs in detector technology ${ }^{3}$. In order to exploit the full capacity of future THz telescope space missions (e.g. Origins Space Telescope ${ }^{4}$ ), new concepts of $\mathbf{T H z}$ coherent receivers are needed, providing larger bandwidths and imaging capabilities with multi-pixel focal plane heterodyne arrays ${ }^{5}$. Here we show that graphene, uniformly doped to the Dirac point, enables highly sensitive and wideband coherent detection of $\mathbf{T H z}$ signals. With material resistance dominated by quantum localization, and thermal relaxation governed by electron diffusion, proof-of-concept graphene bolometers demonstrate a gain bandwidth of $8 \mathrm{GHz}$ and a mixer noise temperature of $475 \mathrm{~K}$, limited by residual thermal background in our setup. An optimized device will result in a mixer noise temperature as low as $36 \mathrm{~K}$, with the gain bandwidth exceeding $20 \mathrm{GHz}$, and a Local Oscillator power of $<100 \mathrm{pW}$. In conjunction with the emerging quantum-limited amplifiers at the intermediate frequency $^{6}$, our approach promises quantum-limited sensing in the THz domain, potentially surpassing superconducting technologies, particularly for large heterodyne arrays. 
Observations in the terahertz (or the far-infrared) frequency range $(\sim 100 \mathrm{GHz}-10 \mathrm{THz})$ are of great importance for understanding physics and chemistry in the star and planet forming regions. ${ }^{7,8}$ With the wavelength in $\mathrm{THz}$ range much longer than that in the IR, terahertz waves have capacity to reveal processes hidden behind dusty gas clouds. Space born telescopes allow overcoming severe atmospheric absorption, whereas a diverse park of instrumentation permits to cover a wide range of scientific tasks. To recover information carried by faint celestial signals, THz frequency mixers -the core of coherent detection- have to fulfill stringent requirements on both sensitivity and, more importantly, on bandwidth, to enable line surveys and studying Doppler-stretched molecular lines. Superconducting hotelectron bolometer (HEB) mixers form the baseline for modern astronomical receivers above $1 \mathrm{THz}$. In these, the wave beating between the incoming $\mathrm{THz}$ signal and detuned local oscillator (LO) causes temperature oscillations at the intermediate frequency (IF), enabling read-out through changes in electrical resistance $R$ (resistive read-out) as long as the temperature in the material can follow the signal modulation. The upper limit for the IF frequency is determined by either the electron-phonon relaxation time $\tau_{e-p h}$ (phonon cooling) or by the out-diffusion time $\tau_{D}=L^{2} /\left(\pi^{2} D\right)$ of hot electrons from the superconductor into the cold electrical contacts (diffusion cooling), with $L$ the length of the device and $D$ the diffusion constant ${ }^{9-11}$. Despite great efforts, $\mathrm{NbN}$ superconducting HEB mixers are limited in terms of instantaneous bandwidth to $\sim 4-5 \mathrm{GHz}^{3}$. Higher bandwidths are possible in new superconductors $^{12}$, but at the expense of forbiddingly high LO power requirements, which is particularly detrimental for array applications. New materials and concepts are needed to go beyond few-pixel THz detectors, available today, to the large-format detector arrays required to enable further cosmic quests ${ }^{4,5,7}$.

In this paper we explore the resistive read-out of the bolometric response of graphene, where quantum effects introduce the long-sought-after temperature dependence of graphene's 
resistance $^{13-15}$. Both long phase coherence times and poor screening of carriers in twodimensions favor quantum interference and electron-electron interactions ${ }^{16-18}$. These effects introduce logarithmic-in-temperature $T$ dependence of the conductivity $\sigma$ of graphene, of the order of $\sigma_{1} \sim e^{2} / h \approx 3.9 \times 10^{-5} \mathrm{~S}$ :

$$
\sigma(\mathrm{T})=\sigma_{0}+\sigma_{1} \ln (T / 1 K)
$$

With graphene at high carrier density $n$, these effects are relatively small. Yet, as $n$ approaches the Dirac point, its conductance $\sigma_{0}$ decreases, and the relative contribution of quantum effects grows. This scenario, appealing for the use of graphene as a thermometer, can however be spoiled by charge disorder. Close to charge-neutrality, disorder leads to doping inhomogeneity, resulting in charge puddles which can effectively shunt quantum transport and cause the resistance of graphene to saturate at low temperatures ${ }^{19-21}$. In contrast, for high quality graphene such as flakes encapsulated by boron nitride ${ }^{22,23}$, reduced disorder results in monotonically increasing resistance at low temperatures. As we recently demonstrated $^{24}$, a similar physical situation occurs when epitaxial graphene on $\mathrm{SiC}$ is brought close to the Dirac point by chemical doping. The spontaneous accumulation of assembly of molecular dopants 2,3,5,6-tetrafluoro-tetracyano-quino-dimethane (F4TCNQ) on graphene via diffusion through poly (methyl-methacrylate) (PMMA) results in a molecular adlayer that dopes epitaxial graphene to the vicinity of the Dirac point and flattens charge inhomogeneity, translating into an effectively reduced disorder. As a result, eq. (1) holds even when graphene is doped to the Dirac point $\left(n<5 \times 10^{9} \mathrm{~cm}^{-2}\right)$ (Fig. 1a), arising primarily from quantum interference in the presence of strong intervalley scattering (Fig. 1b) ${ }^{16,22}$, thus making this material an effective thermometer down to $T=0.2 \mathrm{~K}$. 

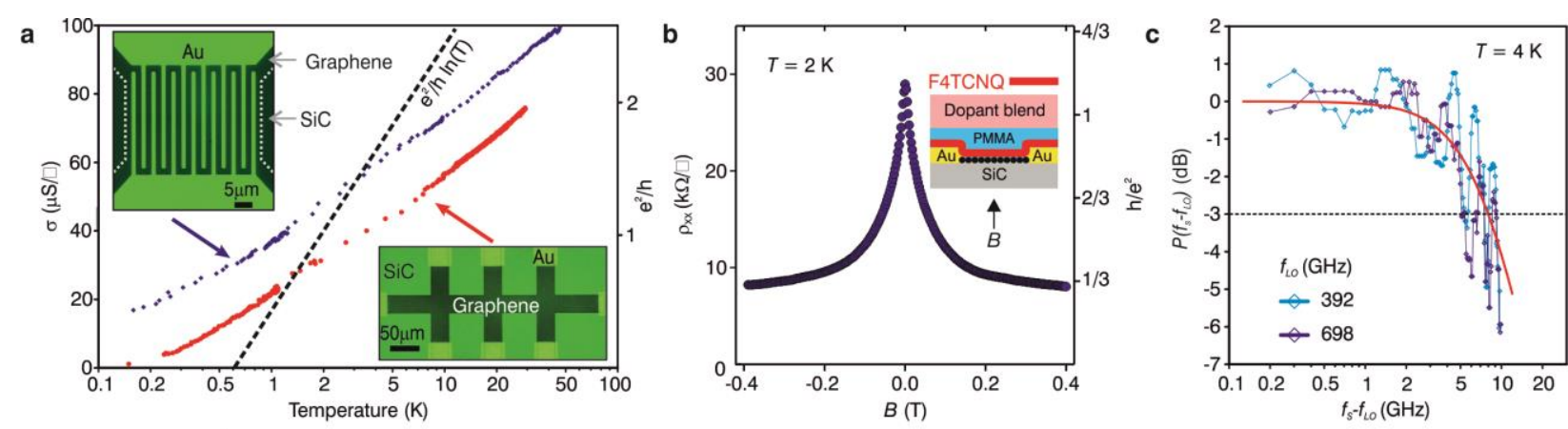

d

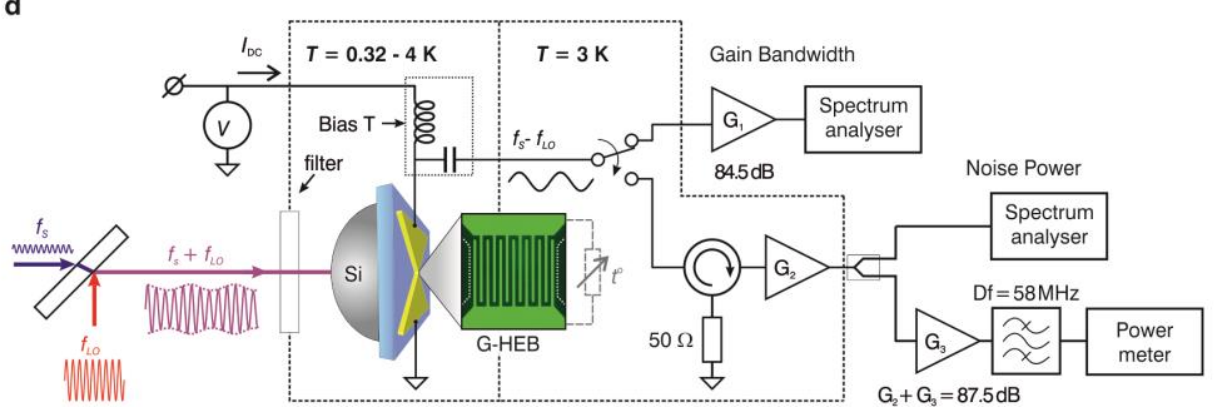

Fig. 1. Graphene doped to the Dirac point as bolometric mixer. b, Zero bias differential conductivity $\sigma(T)=d I /\left.d V\right|_{V=0}$, measured in a dark (optically tight) cryostat for a Hall bar (red) and the $\mathrm{THz}$ mixer (blue), demonstrating logarithmic temperature dependence $\sigma(\mathrm{T})=$ $\sigma_{0}+\sigma_{1} \ln (T / 1 K)$. The dashed line indicates slope $\sigma_{1}=\mathrm{e}^{2} / \mathrm{h}$. b, Suppression of quantum interference effects by perpendicular magnetic field $B$ breaking time reversal symmetry (inset shows the cross-section of the device). c, The gain bandwidth of $8 \mathrm{GHz}$ for our graphene mixer, extracted via measurements of the power at the intermediate frequency $P_{I F}$ as a function of detuning frequency between the signal $f_{s}$ and the local oscillator $f_{L O}$. The solid line is a fit to $P\left(f_{I F}\right)=1 /\left[1+\left(f_{I F} / f_{0}\right)^{2}\right]$, the dashed line indicates $P\left(f_{I F}\right)=-3 \mathrm{~dB}$. Resonances are due to interferences in the optical path and the IF chain. d, Schematics of the experimental setup: the terahertz signal at frequency $f_{s}$ is combined with a monochromatic wave emitted by a local oscillator at a nearby frequency $f_{L O}$, and both are fed into graphene via an integrated bow-tie antenna through a silicon lens.

But, from the point of view of bolometric mixer operation, how fast and sensitive can this thermometer be, while based on charge-neutral graphene? Dependence (1) implies a diverging sensitivity of the resistive read-out $\boldsymbol{d R} / \boldsymbol{d T} \sim \boldsymbol{T}^{-1} \boldsymbol{l n}^{-2}(\boldsymbol{T})$, favoring measurements at low temperatures. The low heat capacity of graphene translates into fast device operation ${ }^{25}$ as long as a proper heat-link is provided. However, the electron-phonon cooling time $\boldsymbol{\tau}_{\boldsymbol{e}-\boldsymbol{p h}} \sim \boldsymbol{n}^{-\mathbf{0 . 5}} \boldsymbol{T}^{-\mathbf{2}}$, diverges at charge neutrality and low temperature ${ }^{26,27}$. Therefore, other cooling pathways, such as electron diffusion cooling, will be required. Although the residual charge puddles do not shunt quantum interference effects, the exact chemical potential 
landscape and microscopic scattering details are not known for the studied material, and these may greatly modify charge and heat transport of graphene at the Dirac point ${ }^{28-31}$. Summarizing, it is a priori not clear how quantum interference and strong electron-electron interactions in epitaxial graphene, chemically doped to the vicinity of the Dirac point, will affect the device speed and noise - it has to be tested experimentally.

We conducted $\mathrm{THz}$ mixing experiments to unveil both the response time and mixer sensitivity in epitaxial graphene chemically doped to the Dirac point. Figure 1c, the central result of this experiment, shows a mixing gain bandwidth of $f_{0}=8 \mathrm{GHz}$, corresponding to a time constant of $1 /\left(2 \pi f_{0}\right)=20$ ps (for temperature independence of gain bandwidth see fig. S1). As we elaborate below, the high Fermi velocity in graphene and highly transparent metallic contacts ${ }^{32}$ in our device allow the heat load in the device to be quickly dissipated by hot electrons diffusing into the metallic leads. To match the resistance of the graphene sample to both the THz impedance of the antenna and the impedance of the IF readout amplifier, we used interdigitated electrodes, with a graphene length of $L=1.5 \mathrm{um}$ and a device width of $W=345 \mathrm{um}$, resulting in nominally 230 squares of graphene in parallel. On-chip Hall bar devices $(L=180 \mu \mathrm{m} \times W=30 \mu \mathrm{m})$ display similar conductivity and temperature dependence $\sigma(\mathrm{T})=\sigma_{0}+\sigma_{1} \ln (T / 1 K)$, confirming our device retains the properties of the 'bulk' chemically doped graphene (Fig. 1a, fig. S2). Figure 1d shows a schematic of our setup, which simultaneously allows for direct current (DC), $\mathrm{THz}$ response, and noise thermometry measurements down to $T=0.32 \mathrm{~K}$ (see also fig. S3). Thorough calibration of the $\mathrm{THz}$ power reaching graphene and the gain of the $\mathrm{GHz}$ chain was performed using noise thermometry (fig. S4, S5).

Remarkably, the DC transport characteristics, and both the $\mathrm{THz}$ direct and mixing response of our device -with transport properties dominated by quantum effects- can all be understood within a simple thermal model of diffusion cooling of electrons. In essence, the 
effect of $\mathrm{THz}$ radiation on our device is that of a temperature increase, with a diffusion cooling rate dominating over phonon cooling of hot electrons (see Supplementary Section 1 and fig. S6). Figure 2 a shows the differential resistance $(d V / d I)$ of the device, measured in the optical cryostat with the $\mathrm{THz}$ source $\mathrm{OFF}$, as a function of the bias current at different temperatures. Complementary, Fig.2b shows $d V I d I$ of the device at the base temperature $T_{0}=0.32 \mathrm{~K}$ for increasing $\mathrm{THz}$ power. In both figures, solid lines correspond to calculations within a Diffusion Cooling Model (DCM), which considers that the heat induced by both DC current and $\mathrm{THz}$ radiation is transferred by charge carriers into the metallic leads kept at the cryostat temperature $T_{0}$ (see also fig. S6, S7 and Supplementary Section 2). Our model assumes the validity of Wiedeman-Franz law and considers that, for temperatures below $6 \mathrm{~K}$, phonon cooling of electrons can be neglected. For the particular case of logarithmic temperature dependence of the bridge conductance, the device current-voltage characteristic $I(V)$ under a THz power $P_{a c}$ can be obtained within the DCM as a recursive expression:

$$
I(V)=G\left(T_{0}\right) V+G_{1} V\left(\frac{\sqrt{1+u^{2}}}{u} \ln \left(\sqrt{1+u^{2}}+u\right)-1\right)
$$

with $u=\frac{1}{V_{T}} \sqrt{V^{2}+\frac{V}{I} P_{a c}}$ and $V_{T}=\sqrt{\mathcal{L}} \cdot T_{0}$. Here, $G(\mathrm{~T})=G_{0}+G_{1} \ln (T / 1 K)$ is the experimentally determined temperature-dependent conductance, yielding $G_{0}=6.55 \mathrm{mS}$ and $G_{1}=3.50 \mathrm{mS}$. From the family of differential resistance curves measured with the $\mathrm{THz}$ source OFF in Fig. 2a, we extract as a fitting parameter the radiation background power $P_{B k g}=0.28 \mathrm{nW}$ in our cryostat, which leads to apparent saturation of conductance at low temperatures (Fig. 2a, inset) . Additionally, the fit returns the Lorenz number $\mathcal{L}=3.1 \times 10^{-8}$ $\mathrm{W} \Omega / \mathrm{K}^{2}$ remarkably close to its theoretical value, $\mathcal{L}=\pi^{2} / 3\left(k_{B} / e\right)^{2} \approx 2.44 \times 10^{-8} \mathrm{~W} \Omega / \mathrm{K}^{2}$, despite graphene being at the charge neutrality point ${ }^{33}$. Next, we use the same $P_{B k g}$ and $\mathcal{L}$ to predict a set of curves for the $d V / d I$ response under $\mathrm{THz}$ irradiation at base temperature 
$T_{0}=0.32 \mathrm{~K}$, considering that the total absorbed $\mathrm{THz}$ power $\left(P_{a c}\right)$ includes contribution from both the LO and $P_{B k g}$. Figure $2 \mathrm{~b}$ shows, with no extra fitting parameters involved, that the DCM precisely reproduces the observed bolometric response to radiation.
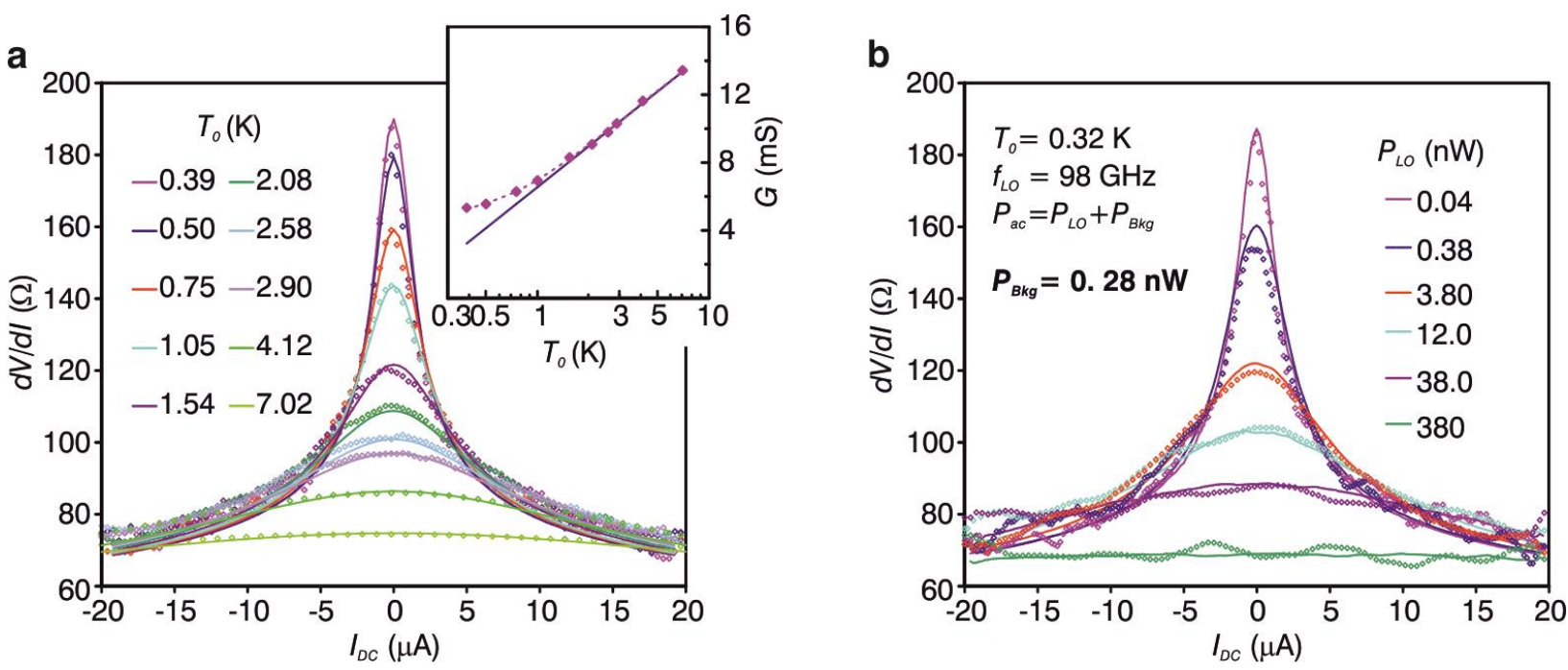

Fig. 2. Equivalence of $\mathrm{THz}$ radiation, Joule heating and base temperature effects on the graphene bolometer. a, Effect of temperature on the measured differential resistance $d V / d I(I)$ with the $\mathrm{THz}$ source OFF. Inset: Zero bias conductance for the same device. Deviation from $G(\mathrm{~T})=G_{0}+G_{1} \ln (T / 1 K)$ at low temperature, absent in measurements in a dark cryostat (Fig. 1A), is due to background heat load $P_{B k g}$. b, Effect of THz power $\left(f_{L O}=\right.$ $98 \mathrm{GHz}$ ) on the $d V / d I$ response of our device at $T_{0}=0.32 \mathrm{~K}$. In both cases dots correspond to measurements and solid lines to the DCM prediction.

Having established the bolometric nature of the $\mathrm{THz}$ response in our device, we investigate its performance as a THz mixer. We irradiated the device with two THz sources, a signal S, and the LO, measuring the mixer gain $G_{m i x}=P_{I F} / P_{S}$ which is the ratio of the power at the output $\left(P_{I F}\right)$ to the incoming signal power $P_{s}($ Fig. 3A). Solid lines in Fig. 3a show that, within all of our tested parameter space $\left(T_{0}, P_{L O}, I_{D C}\right), G_{m i x}$ is well described by the classical bolometric mixer expression ${ }^{34}$, which also follows from the DCM (for details of the DCM, see Supplementary Section 2):

$$
G_{\operatorname{mix}}=\frac{1}{2} \frac{P_{L O}}{P_{D C}} \frac{50 \Omega}{V / I}\left[\frac{1-\left(\frac{V}{I}\right)\left(\frac{d I}{d V}\right)}{1+50 \Omega\left(\frac{d I}{d V}\right)}\right]^{2}
$$


The maximum mixer gain is $G_{m i x}=-27 \mathrm{~dB}\left(P_{I F} / P_{S}=2 \%\right)$ at optimum mixing conditions $\left(P_{L O}=3.8 \mathrm{nW}, I_{D C}=5 \mu \mathrm{A}\right)$. At this operating point, the electronic temperature in graphene, measured by noise thermometry, is elevated above the base temperature due to the DC bias, the LO, and background radiation powers, reaching an effective noise equivalent temperature $T_{S} \approx 1.9 \mathrm{~K}$, as shown in Fig. $3 \mathrm{~b}$. Solid lines in Fig. $3 \mathrm{~b}$ correspond to the DCM considering that the microwave noise of graphene is purely thermal (Johnson noise) (see also Supplementary Section 3 and fig. S8). For a coherent mixer, the ultimate sensitivity is determined by its noise temperature $T_{m i x}$, and for our device we find $T_{\text {mix }}=T_{S} /\left(2 G_{\text {mix }}\right)=475 \mathrm{~K}$ (a factor 2 accounts for the double sideband nature of coherent mixing).
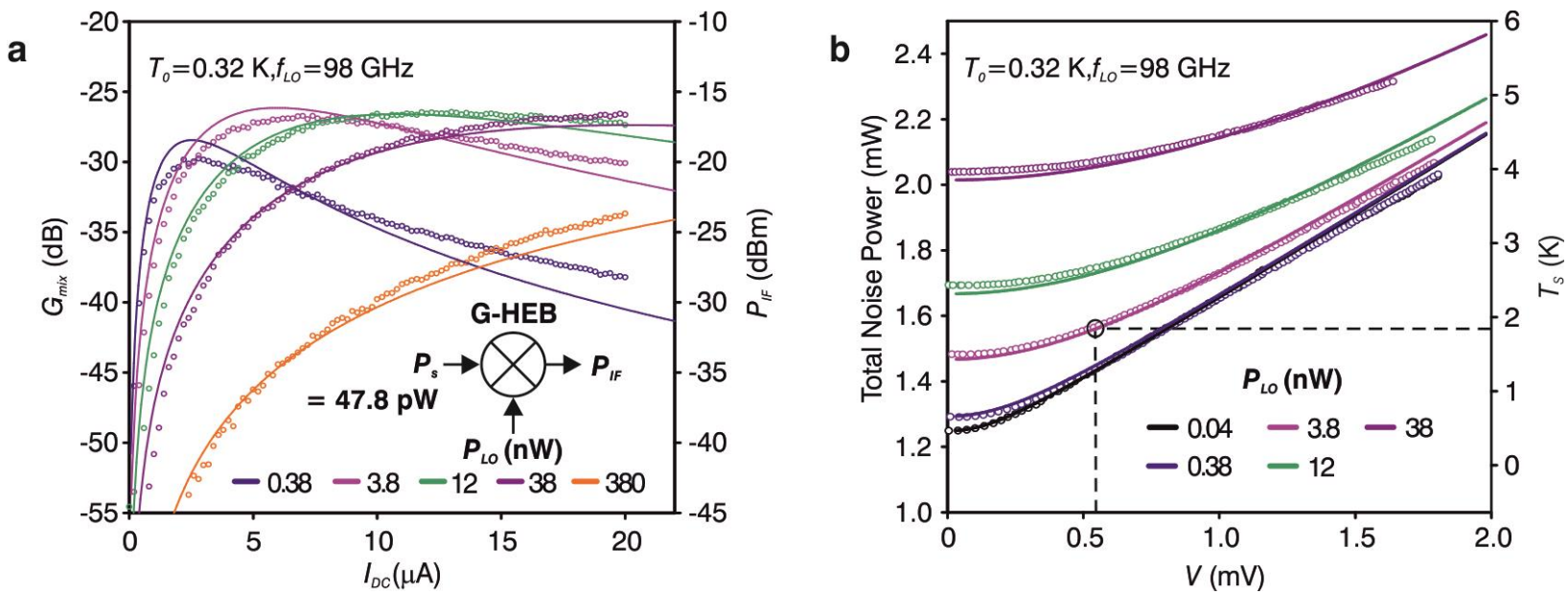

Fig. 3. Terahertz mixing performance of our graphene bolometer. a, Mixing gain $G_{m i x}$ $\left(f_{I F}=3.5 \mathrm{GHz}, P_{S}=47.8 \mathrm{pW}\right)$ and $\mathbf{b}$, Thermal noise power measurements for different $\mathrm{LO}$ power levels $P_{L O}\left(f_{I F}=4 \mathrm{GHz}\right)$. Dots correspond to measurements. With a radiation background $P_{B k g}=0.5 \mathrm{nW}$, solid lines in (a) correspond to eq. (2) and the right y-axis is the measured output power at the IF. In $\mathbf{b}$, solid lines are fits to the DCM, and the right y-axis displays the graphene noise equivalent temperature $T_{S}$, calculated with the DCM.

With the validity of DCM demonstrated, we extrapolate the intrinsic performance of our device for zero background radiation conditions $\left(P_{B k g}=0\right)$, as expected on a space-born mission. Figure 4a shows that zero background allows for operation at reduced DC bias, resulting in the increase of the achievable mixing gain $G_{m i x}\left(I_{D C}, P_{L O}\right)$ by a few dB compared to the gain we measured in our cryostat (Fig. 3A). Combining the mixer gain and the $T_{S}$ 
corresponding to maximum conversion conditions, we show the calculated mixer noise temperature as a function of the base temperature $T_{0}$ in Fig. $4 \mathrm{~b}$ (see also Supplementary Section 4 and fig. S9). Under these considerations, cooling the device down to $T_{0}=0.2 \mathrm{~K}$, a limit set by the validity of logarithmic fit in our model and achievable in refrigerators for satellite missions ${ }^{35}$, leads to $T_{m i x}=36 \mathrm{~K}$. Remarkably, this implies that the graphene bolometer mixer will operate in a quantum-limited regime at all frequencies above $k_{B} T_{\text {mix }} / h=0.75 \mathrm{THz}$.
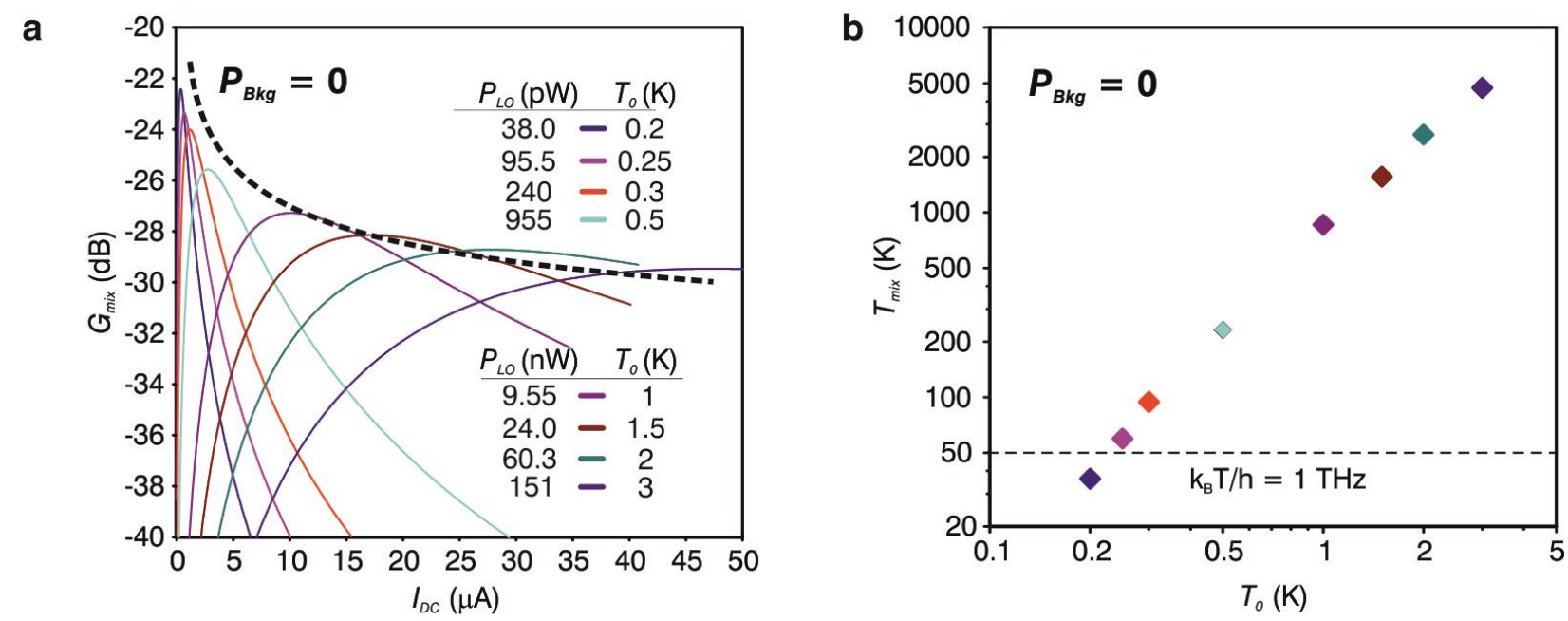

Fig. 4. Intrinsic performance of the graphene bolometric mixer (without background radiation). a, Mixing performance for different operating temperatures $T_{0}$. For each temperature the curve corresponding to an optimum LO power (highest possible gain) is shown. Dashed line represents an approximated expression for the mixing gain $G_{m i x} \propto$ $\left(G_{1} V / I\right)^{2}$. b, Mixer noise temperature $\left(T_{\text {mix }}=T_{\text {noise }} / 2 G_{m i x}\right)$ under optimal conditions as a function of the base temperature $T_{0}$.

The performance of our $\mathrm{THz}$ mixer improves drastically at lower temperatures, and thus can take full advantage of space-oriented cryocoolers providing sub-Kelvin temperatures ${ }^{35}$. In our experiments the graphene temperature at optimum operation conditions was limited by the background radiation power. At zero radiation background, we expect $T_{S} \sim 0.4 \mathrm{~K}$; combined with the best available commercial amplifiers $\left(T_{A m p} \sim 1 \mathrm{~K}\right)$, this translates into a mixing noise figure of $125 \mathrm{~K}$ for the mixer-amplifier chain. Unleashing the full potential of the presented mixer requires a wideband amplifier with sub-Kelvin noise. Such devices are 
emerging on the wave of progress in quantum technologies ${ }^{6}$. For an IF amplifier with an added noise of about one photon at $10 \mathrm{GHz}$, the mixer input-referred noise will be $1 / G_{\text {mix }} \approx$ 100 photons, or $\sim 1$ in units of $1 \mathrm{THz}$ photons.

Our results are based on our ability to decrease the carrier density in graphene without degrading its transport properties. In this scenario, quantum transport in graphene at the Dirac point enables highly sensitive and broadband terahertz coherent mixing. Currently demonstrating a gain bandwidth of $8 \mathrm{GHz}$ for a $L=1.5 \mu \mathrm{m}$ long device, and with its operation fully described by a diffusion cooling model, we expect the bandwidth of the device to scale as $\sim L^{-2}$ (see also Supplementary Section 1 and fig. S6) ${ }^{9-11}$. Thus, for a device length of $0.8-1 \mu \mathrm{m}$ (attainable with current microfabrication technologies), the bandwidth can in principle be extended to $20 \mathrm{GHz}$, far superior to what is achievable in superconducting mixers. Combining wafer-scale epitaxial graphene on $\mathrm{SiC}$ and sub-nW LO power requirements, this mixing platform is appealing for implementing large focal plane arrays of THz mixers. Finally, the achievable mixing gain $G_{\text {mix }} \propto\left(G_{1} V / I\right)^{2}$ is set by the strength of quantum effects, which in our material are quantified as $\sigma_{1} \approx 0.3 e^{2} / h$ (Fig. 1a). Further efforts will unveil if a different combination of polymers and molecular dopants can lead to an increased $\sigma_{1}$, as a result of an even more homogeneous doping closer to the Dirac point or by tuning a particular interplay of microscopic scattering mechanisms.

\section{References:}

1. Rowan-Robinson, M. Astronomy. Probing the cold universe. Science 325, 546-7 (2009).

2. Young, E. T. et al. Early science with SOFIA, the stratospheric observatory for infrared astronomy. Astrophys. J. Lett. 749, L17 (2012).

3. de Graauw, T. et al. The Herschel -Heterodyne Instrument for the Far-Infrared (HIFI). 
Astron. Astrophys. 518, L6 (2010).

4. Battersby, C. et al. The Origins Space Telescope. Nature Astronomy 2, 596-599 (2018).

5. Goldsmith, P. F. Sub-Millimeter Heterodyne Focal-Plane Arrays for High-Resolution Astronomical Spectroscopy. Radio Sci. Bull. 362, 53-73 (2017).

6. Ho Eom, B., Day, P. K., Leduc, H. G. \& Zmuidzinas, J. A wideband, low-noise superconducting amplifier with high dynamic range. Nat. Phys. 8, 623-627 (2012).

7. Blain, A. W., Smail, I., Ivison, R. J., Kneib, J. P. \& Frayer, D. T. Submillimeter galaxies. Physics Report 369, 111-176 (2002).

8. Madau, P. \& Dickinson, M. Cosmic Star Formation History. Annu. Rev. Astron. Astrophys. 52, 415-486 (2014).

9. Klapwijk, T. M. \& Semenov, A. V. Engineering Physics of Superconducting HotElectron Bolometer Mixers. IEEE Trans. Terahertz Sci. Technol. 7, 627-648 (2017).

10. Prober, D. E. Superconducting terahertz mixer using a transition-edge microbolometer. Appl. Phys. Lett. 62, 2119-2121 (1993).

11. Gershenzon, E. M. et al. Millimeter and submillimeter range mixer based on electronic heating of superconducting films in the resistive state. Sov. Phys. Supercond. 3, 15821597 (1990).

12. Novoselov, E. \& Cherednichenko, S. Low noise terahertz MgB2 hot-electron bolometer mixers with an $11 \mathrm{GHz}$ bandwidth. Appl. Phys. Lett. 110, 032601 (2017).

13. El Fatimy, A. et al. Epitaxial graphene quantum dots for high-performance terahertz bolometers. Nat. Nanotechnol. 11, 335-8 (2016).

14. Cai, X. et al. Sensitive room-temperature terahertz detection via the 
photothermoelectric effect in graphene. Nat. Nanotechnol. 9, 814-819 (2014).

15. Mittendorff, M. et al. Ultrafast graphene-based broadband THz detector. Appl. Phys. Lett. 103, (2013).

16. McCann, E. et al. Weak-Localization Magnetoresistance and Valley Symmetry in Graphene. Phys. Rev. Lett. 97, 14-17 (2006).

17. Aleiner, I. L. \& Efetov, K. B. Effect of disorder on transport in graphene. Phys. Rev. Lett. 97, 236801 (2006).

18. Lara-Avila, S. et al. Disordered Fermi Liquid in Epitaxial Graphene from Quantum Transport Measurements. Phys. Rev. Lett. 107, 166602 (2011).

19. Novoselov, K. S. et al. Electric field effect in atomically thin carbon films. Science 306, 666-9 (2004).

20. Novoselov, K. S. et al. Two-dimensional gas of massless Dirac fermions in graphene. Nature 438, 197-200 (2005).

21. Zhang, Y., Tan, Y.-W., Stormer, H. L. \& Kim, P. Experimental observation of the quantum Hall effect and Berry's phase in graphene. Nature 438, 201-4 (2005).

22. Ponomarenko, L. A. et al. Tunable metalinsulator transition in double-layer graphene heterostructures. Nat. Phys. 7, 958-961 (2011).

23. Efetov, D. K. et al. Fast thermal relaxation in cavity-coupled graphene bolometers with a Johnson noise read-out. Nature Nanotechnology 1-5 (2018).

24. He, H. et al. Uniform doping of graphene close to the charge neutrality point by polymer-assisted spontaneous assembly of molecular dopants. Nat. Commun. 9, 3956 (2018).

25. Balandin, A. A. Thermal properties of graphene and nanostructured carbon materials. 
Nature Materials 10, 569-581 (2011).

26. Kubakaddi, S. S. Interaction of massless Dirac electrons with acoustic phonons in graphene at low temperatures. Phys. Rev. B - Condens. Matter Mater. Phys. 79, 075417 (2009).

27. Baker, A. M. R. et al. Energy loss rates of hot Dirac fermions in epitaxial, exfoliated, and CVD graphene. Phys. Rev. B - Condens. Matter Mater. Phys. 87, 045414 (2013).

28. Narozhny, B. N., Gornyi, I. V., Titov, M., Schütt, M. \& Mirlin, A. D. Hydrodynamics in graphene: Linear-response transport. Phys. Rev. B - Condens. Matter Mater. Phys. 91, 035414 (2015).

29. Foster, M. S. \& Aleiner, I. L. Slow imbalance relaxation and thermoelectric transport in graphene. Phys. Rev. B 79, 085415 (2009).

30. Müller, M., Fritz, L. \& Sachdev, S. Quantum-critical relativistic magnetotransport in graphene. Phys. Rev. B 78, 115406 (2008).

31. Crossno, J. et al. Observation of the Dirac fluid and the breakdown of the WiedemannFranz law in graphene. Science 351, 1058-1061 (2016).

32. Wang, L. et al. One-Dimensional Electrical Contact to a Two-Dimensional Material. Science 342, 614-617 (2013).

33. Crossno, J. et al. Observation of the Dirac fluid and the breakdown of the WiedemannFranz law in graphene. Science 351, 1058-61 (2016).

34. Ekström, H., Karasik, B. S., Kollberg, E. L. \& Yngvesson, K. S. Conversion Gain and Noise of Niobium Superconducting Hot-Electron-Mixers. IEEE Trans. Microw. Theory Tech. 43, 938-947 (1995).

35. Martin, F., Vermeulen, G., Camus, P. \& Benoit, A. A closed cycle3He-4He dilution 
refrigerator insensitive to gravity. in Cryogenics 50, 623-627 (Elsevier, 2010).

Acknowledgments: We thank Alexander Tzalenchuk, Justin F. Schneiderman and Tord Claeson for useful discussion and critical reading of the manuscript. This work was jointly supported by the Swedish Foundation for Strategic Research (SSF) (No. IS14-0053, GMT140077, RMA15-0024), Knut and Alice Wallenberg Foundation, Chalmers Area of Advance NANO, the Swedish Research Council (VR) 2015-03758 and 2016-048287, the SwedishKorean Basic Research Cooperative Program of the NRF (No. NRF2017R1A2A1A18070721), European Union's Horizon 2020 research and innovation programme (grant agreement No 766714/HiTIMe).

Author contributions: S.L.A., H.H., K.H.K. and R.Y contributed to sample growth and device fabrication. S.L.A., H.H., K.H.K, F.L. and T.B. performed the DC characterization of the device. D.G. developed the theoretical calculations. A.D. and S.Ch. characterized the sample at $\mathrm{THz}$ and microwave frequency ranges. D.G., S.L.A, A.D., S.Ch and S.K contributed to the interpretation of the experiments. S.K., S.L.A and S.Ch. conceived and designed the experiment. All the authors contributed to the writing of the manuscript.

Competing interests: Authors declare no competing interests.

Materials \& Correspondence: should be addressed to samuel.lara@chalmers.se

Data and materials availability: The authors declare that the main data supporting the findings of this study are available within the article and its Supplementary Information files. Additional data is available from the corresponding author upon request. 


\section{Methods}

\section{$\underline{\text { Sample Fabrication }}$}

Monolayer $\mathrm{SiC} / \mathrm{G}$ was grown on the $\mathrm{Si}$-face of $\mathrm{SiC}$ using thermal decomposition of $7 \mathrm{~mm} \times 7$ $\mathrm{mm} \mathrm{SiC} \mathrm{substrates.} \mathrm{The} \mathrm{samples} \mathrm{were} \mathrm{grown} \mathrm{in} \mathrm{argon} \mathrm{atmosphere} 800$ mbar, and at a temperature of around $1700{ }^{\circ} \mathrm{C}$ for $5 \mathrm{~min}$. Optical microscopy reveal a typical surface coverage of $>95 \%$ monolayer graphene. Devices were fabricated using conventional electron beam lithography, using only PMMA resist (Microlithography Chemicals Corp.) bilayer and lift-off. The electrical contacts were fabricated using physical vapor deposition of $\mathrm{Ti} / \mathrm{Au}, 5 \mathrm{~nm}$ and $80 \mathrm{~nm}$ thick respectively. After fabrication, samples were cleaned using isopropyl alcohol, acetone and dried using nitrogen gas. After this step, the samples were spin-coated with a $100 \mathrm{~nm}$-thick PMMA layer (Microlithography Chemicals Corp.), followed by spin coating of a $170 \mathrm{~nm}$-thick chemical dopant blend. The dopant blend consists of a mixture of F4TCNQ (Sigma-Aldrich) and PMMA (Microlithography Chemicals Corp.). In detail, $25 \mathrm{mg}$ of dry F4TCNQ powder is mixed with $3 \mathrm{ml}$ anisole solvent. Subsequently, 0.5 $\mathrm{ml}$ of this solution is then mixed with $1 \mathrm{ml}$ PMMA A6 (6\% PMMA by weight in anisole). The resulting ratio of PMMA to FT4CNQ is roughly 93:7 by weight. All polymer layers are deposited on graphene using spin coating at $6000 \mathrm{rpm}$ for $1 \mathrm{~min}$. A 5 min baking step on a hotplate at $160{ }^{\circ} \mathrm{C}$ follows each spin coating step. This process consistently yields a carrier density $|n|<1 \times 10^{10} \mathrm{~cm}^{-2}$ and sheet resistance of about $\rho=25 \mathrm{k} \Omega / \mathrm{sq}$ at $T=2 \mathrm{~K}$.

Gain Bandwidth measurements

Frequency mixing experiments were done with the two-wave mixing approach, where the frequency of one $\mathrm{THz}$ source is kept constant, while the frequency of the second source is changed. The resulting $\mathrm{THz}$ current through the device becomes amplitude modulated with the beating frequency $\left(f_{I F}=\left|\mathrm{f}_{\mathrm{LO}}-\mathrm{f}_{\mathrm{S}}\right|\right)$. The output signal from the device under test is amplified and its power is measured with a spectrum analyzer. Measurements were conducted with $\mathrm{THz}$ generators in all three frequency bands 100, 300 and $700 \mathrm{GHz}$. For broadband measurements, with the beating frequency $f_{I F}$ at least up to $10 \mathrm{GHz}$, both the bias$\mathrm{T}$ and the amplifiers were held at room temperature. Gain calibration for the readout chain was conducted separately. The only factors which were not included in the calibration were the effect of the mixer block and the $\mathrm{THz}$ standing waves in the optical path. These residuals lead to some ripples in the measured signal. The fitting curve $P\left(f_{I F}\right)=1 /\left[1+\left(f_{I F} / f_{0}\right)^{2}\right]$ in Fig. $1 \mathrm{C}$ of the main text provides a roll-off frequency of $f_{0}=8 \mathrm{GHz}$. 